Urologe $2019 \cdot 58: 1498$

https://doi.org/10.1007/s00120-019-01076-w Online publiziert:27. November 2019

(c) Springer Medizin Verlag $\mathrm{GmbH}$, ein Teil von Springer Nature 2019

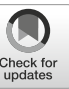

T. Bschleipfer' $\cdot$ R. Doggweiler ${ }^{2}$ D. Schultz-Lampel ${ }^{3} \cdot$ J. de Jong ${ }^{4} \cdot$ A. Gonsior ${ }^{5}$. J. Hensen ${ }^{6} \cdot$ E. Heßdörfer ${ }^{7}$ • B. T. Kaftan ${ }^{8} \cdot$ A. Kuhn ${ }^{9}$ U. Kunzendorf ${ }^{10} \cdot$ A. Lampel ${ }^{11}$.

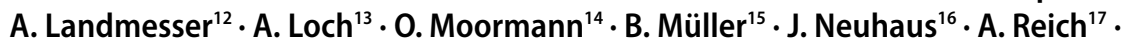
R. Roth ${ }^{18} \cdot$ S. Schumacher ${ }^{19} \cdot$ R. Stratmeyer ${ }^{20} \cdot$ W. Vahlensieck ${ }^{21} \cdot$ A. Wördehoff ${ }^{22}$. B. Münder-Hensen ${ }^{23}$

${ }^{1}$ Klinik für Urologie, Andrologie und Kinderurologie, Interdisziplinäres Kontinenz- und Beckenbodenzentrum, Zentrum für Interstitielle Cystitis und Beckenschmerz, Klinikum Weiden/Kliniken Nordoberpfalz AG, Weiden i.d.OPf., Deutschland; ${ }^{2}$ Kontinenzzentrum Hirslanden, Zürich, Schweiz; ${ }^{3}$ Kontinenzzentrum Süd-West, Schwarzwald-Baar-Klinikum, Villingen-Schwenningen, Deutschland; ${ }^{4}$ Physio Artos, Interlaken, Schweiz; ${ }^{5}$ Klinik für Urologie, Universität Leipzig AöR, Leipzig, Deutschland; ${ }^{6}$ MICA Multinational Interstitial Cystitis Association, Euskirchen, Deutschland; ${ }^{7}$ Blasenzentrum Westend, Urologische Privatarztpraxis, Berlin, Deutschland; ${ }^{8}$ Städtisches Klinikum Lüneburg, Lüneburg, Deutschland; ' ${ }^{9}$ Zentrum für Urogynäkologie, Frauenklinik, Inselspital Bern, Bern, Schweiz; ${ }^{10} \mathrm{Klinik}$ für Innere Medizin IV, Univers. Klinikum Schleswig-Holstein, Universität Kiel, Kiel, Deutschland; " Klinik für Urologie und Kinderurologie, Schwarzwald-Baar-Klinikum, Villingen-Schwenningen, Deutschland; ${ }^{12}$ Physiohof Landmesser, Erkelenz, Deutschland; ${ }^{13}$ Urologische Praxis, Westerland, Deutschland; ${ }^{14}$ St.-Josefs-Hospital Dortmund-Hörde, Klinik für Urologie, Katholische St. Lukas Gesellschaft, Dortmund, Deutschland; ${ }^{15}$ Urologische Praxis, Berlin-Charlottenburg, Deutschland; ${ }^{16}$ Forschungslabor der Klinik und Poliklinik für Urologie, Universität Leipzig AöR, Leipzig, Deutschland; ${ }^{17}$ Klinik für Frauenheilkunde \& Geburtshilfe, Donauklinik Neu-Ulm, Neu-Ulm, Deutschland; ${ }^{18}$ Kirchheim, Deutschland; ${ }^{19}$ Healthpoint Hospital, Abu Dhabi, Vereinigte Arabische Emirate; ${ }^{20}$ Curos - Urologisches Zentrum in der Klinik Links vom Rhein, Köln-Rodenkirchen, Deutschland; ${ }^{21}$ Urologische Abteilung, Kurpark-Klinik, Bad Nauheim, Deutschland; ${ }^{22}$ ehem. Praxisklinik Wördehoff, Mechernich, Deutschland; ${ }^{23}$ ICA-Deutschland, Euskirchen, Deutschland

\title{
Erratum zu: Diagnostik und Therapie der interstitiellen Zystitis (IC/BPS). S2k-Leitlinie der Deutschen Gesellschaft für Urologie
}

\section{Erratum zu:}

\section{Urologe 2019}

https://doi.org/10.1007/s00120-019-

01054-2

Leider ist in der $\bullet$ Infobox 5 ein Fehler aufgetreten. Korrekt muss es hier „Komplementärmedizinische Therapie - Empfehlungen und Konsens" heißen.

Wir bitten die korrekte Bezeichnung zu beachten und den Fehler zu entschuldigen.

\section{Infobox 5 Komplementärmedi- zinische Therapie - Empfehlungen und Konsens}

Trotz schwer interpretierbarer Studienergebnisse kann durch Akupunktur eine signifikante Reduktion der Scores von VAS, PUF, ICSI erreicht werden [kann erwogen werden - $100 \%$ ].

Eine Mikrobiologische Therapie kann Funktionsstörungen der Schleimhäute und des Immunsystems regulieren [kann erwogen werden $-85 \%$ ].

Laut Theorie kann eine Neuraltherapie Störungen beseitigen, die sich in IC-Symptomatik ausdrücken [kann erwogen werden - $95 \%$ ].

Eine Orthomolekulare Therapie kann Mikronährstoffdefizite ausgleichen [kann erwogen werden - 95\%]

\section{Korrespondenzadresse}

Prof. Dr. med. Dr. phil. T. Bschleipfer, F.E.B.U.

Klinik für Urologie, Andrologie und Kinderurologie, Interdisziplinäres Kontinenzund Beckenbodenzentrum, Zentrum für Interstitielle Cystitis und Beckenschmerz, Klinikum Weiden/Kliniken Nordoberpfalz AG Söllnerstraße 16, 92637 Weiden i.d.OPf., Deutschland thomas.bschleipfer@kliniken-nordoberpfalz.ag

Die Online-Version des Originalartikels ist unter https://doi.org/10.1007/s00120-019-01054-2 zu finden. 